\title{
Research on GDP Forecast of the Inner Mongolia Autonomous Region Based on ARIMA Model
}

\author{
Zihan Zhao ${ }^{1, *}$ \\ ${ }^{1}$ School of Information Qingdao No.2 middle school, Qingdao, Shandong 193000, China \\ *Corresponding author. Email: Isabella03254@163.com
}

\begin{abstract}
This study focuses on the GDP development and forecast of Inner Mongolia Autonomous Region from 1993 to 2020. ARIMA model and time series forecasting are built to analyze the population development and forecast the GDP index in the next five years. The results show that the GDP of Inner Mongolia Autonomous Region will further increase to some extent, and these data can be regarded as a good assistant for the government of Inner Mongolia Autonomous Region in planning the next stage of economic development. And guide the government to further planning and development.
\end{abstract}

Keywords: GDP, ARIMA Model, Inner Mongolia, Time series forecasting

\section{INTRODUCTION}

There are 34 provincial-level administrative regions in the People's Republic of China, among which the Inner Mongolia Autonomous Region is the third largest administrative region. It is also home to the Mongols, the ninth largest of China's 56 ethnic minorities. Because of its landlocked location, bordering Mongolia to the north, its location is also important. According to the table and image, it is not difficult to see that, except for 2017, the GDP of Inner Mongolia Autonomous Region has been increasing year by year and maintained a relatively reasonable and efficient speed. Due to the small base, it cannot be compared with some provinces with comparable territory at present, but it is also developing towards a good place. Over the past 70 years, especially since the reform and opening up, under the leadership of Inner Mongolia Autonomous Region Government, the economy and society of Inner Mongolia have undergone earth-shaking changes. The comprehensive economic strength, industrial structure and scale, infrastructure construction, opening-up and cooperation, and people's living standards of Inner Mongolia have achieved historic leaps forward.

The purpose of this study is to analyze and predict the economy of Inner Mongolia Autonomous Region of China by using time series. We can record the process of GDP change and development according to time series, add serial observation and research of GDP line, find out the law of its change and development, and predict its future trend. Through the time series analysis, we can find out the rule of GDP growth in Inner Mongolia Autonomous Region, so that the time series analysis can play the role of macro-control and microcontrol. Because this theory and practice are constantly developing, through the past GDP forecast and continuous practice test, in order to find problems and effectively correct the problems of the future development of China's Inner Mongolia Autonomous Region GDP as a reference is the significance of this paper. GDP refers to the economic output of a country or region at a certain time. The value of all final products and services. It is often referred to as national economic condition, and its best indicator can reflect not only the economic condition of a country or region, but also the strength and wealth of a country or region. This includes all resident units in the area.

In this paper, the GDP of China's Inner Mongolia Autonomous Region is selected as the data material, the data is processed, the model is established, and the established model is tested, and the determined model is used for the domestic production of China's Inner Mongolia Autonomous Region. The forecast results are then compared with actual GDP. The results show that the predicted time series model is good.

\section{LITERATURE REVIEW}

"Urbanization and Socioeconomic Development in Inner Mongolia in 2000 and 2010: A GIS Analysis" 
expresses the impact of economic indicators and other indicators of overall development on the Inner Mongolia Autonomous Region, and discusses the relationship between urbanization and development. By calculating the Human Development Index (HDI), it compares some of the economic impacts of other regions in China due to the geographical location, and proposes that urbanization is an important factor in the process of using rapid economic growth to improve education and health. ${ }^{[1] " R e s e a r c h ~ o n ~ t h e ~ E c o n o m i c ~ I m p a c t ~ o f ~ N e w ~}$ Energy Fiscal and Tax Policies Based on CGE Model A Case from Inner Mongolia" described that different fiscal and tax subsidies and preferential tax policies can promote the development of Inner Mongolia's wind power and solar photovoltaic power generation industries, and use the dynamic 3 ED-CGE model to influence economy. After a series of studies, the financial subsidies by using the attenuation mechanism can better promote GDP growth, residents' welfare and employment. ${ }^{[2]}$ "Livestock Production in China: An Empirical Study in Inner Mongolia" uses panel data to examine the impact on livestock production, grazing intensity and structure on the field survey of herders in the Inner Mongolia Autonomous Region, and derives a series of results related to grazing through the results of an econometric model. ${ }^{[3]}$ "County-level estimates of population and economic scenarios under the shared socioeconomic pathways: A case study in Inner Mongolia, China" uses PDE and CD models to simulate and study the population and economic changes of counties in the Inner Mongolia Autonomous Region according to different SSP scenarios, according to my country's current population policy and social and economic development in Inner Mongolia. The research results provide technical solutions for population and economic forecasting. ${ }^{[5]}$

"A Comparison of ARIMA and LSTM in Forecasting Time Series"' s research is whether or how newly developed algorithms based on predictive time series data of deep learning, such as "Long Short Term Memory (LSTM)", can outperform traditional algorithms. Research has shown that algorithms based on deep learning are more appropriate than traditional algorithms. ${ }^{[6]}$ "Application of the ARIMA model on the COVID-2019 epidemic dataset" proposed a simple econometric model to help predict the spread of COVID-19 and carried out an ARIMA model based on epidemiological data to predict the epidemiological trend of COVID-19 and further comparisons or look to the future. ${ }^{[7] " C o m p a r i s o n ~ o f ~ A R I M A ~ a n d ~} \operatorname{GM}(1,1)$ models for prediction of hepatitis B in China" performs statistical analysis through ARIMA model and grey model $\operatorname{GM}(1,1)$, showing better hepatitis $b$ fitting and forecasting performance. ${ }^{[8]} \quad$ "Hydroelectricity consumption forecast for Pakistan using ARIMA modeling and supply-demand analysis for the year 2030 " presents a forecasting study based on the historical data which using ARIMA modeling. The research would be quite useful in the management of water resources of Pakistan for future. In "Forecasting Egyptian GDP Using ARIMA Models", the BoxJenkins method is used to construct an appropriate ARIMA model based on the Egyptian GDP data. The study found that the appropriate statistical model for Egypt's GDP is $\operatorname{ARIMA}(1,2,1)$ and used the model to predict Egypt's GDP for the next ten years. ${ }^{[4] " R e s e a r c h}$ on GDP Forecast of Ji'an City Based on ARIMA Model" uses the GDP of Ji'an in the past 40 years and the ARIMA $(0,2,1)$ model to predict the future GDP of Ji'an. The results forecast for the development of Ji'an City economic development aims to offer scientific consult. ${ }^{[9]}$

Figure 1 shows the visual operation flow of the ARIMA modeling steps. First, we need to examine the stationarity to be studied, and the second step is to preprocess the data to check whether it is stable. If the research time series data is non-stationary, the data must be difference operation. Otherwise, continue to the next step. The third part checks whether the stationary time series is a white noise series. If it is, it means that the analysis should be stopped because there is no value; if it is not, you can go to the next step. The fourth step is to select the ARIMA model structure. The fifth step requires specific parameter values in the ARIMA model. The sixth step is to perform a white noise test. If the residual sequence passes the test, it can be terminated; if it does not pass the white noise, the model needs to be modified. The seventh part is to use the finally established ARIMA model to make prediction in a short period of time.

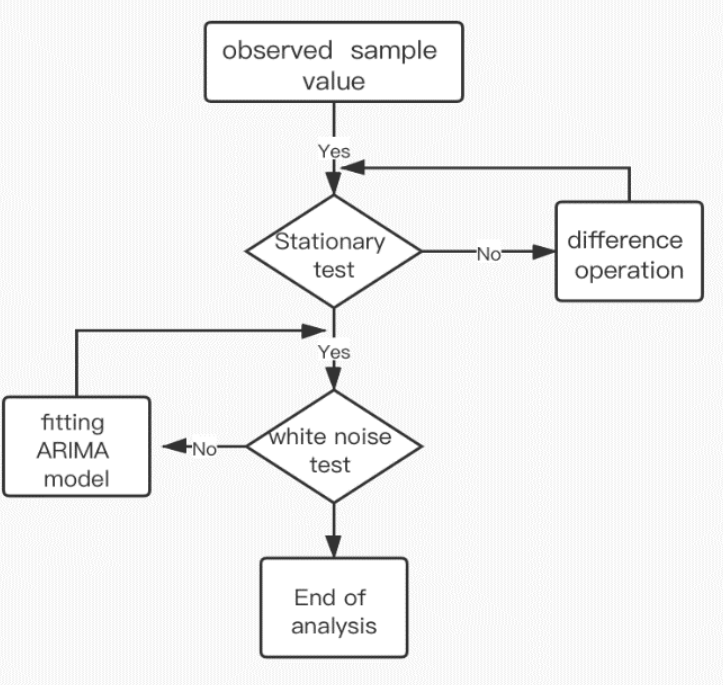

Figure 1 ARIMA modeling process 


\section{RESULTS AND DISCUSSION}

\subsection{Data Description}

This paper takes the gross domestic product statistical data released by the comprehensive data platform of the Bureau of Statistics of the Inner Mongolia Autonomous Region as the data source, selects the GDP data of the Inner Mongolia Autonomous Region from 1993 to 2020 as the sample, establishes the ARIMA model, and forecasts the GDP of the Inner Mongolia Autonomous Region from 2021 to 2025.

\subsection{Stationary Test}

Based on previous data, GDP of the past few years is taken as time series to obtain its change rule, establish a prediction model and forecast Future developments and changes are of great significance. This paper takes the GDP of China's Inner Mongolia Autonomous Region from 1993 to 2020 as an example, uses the time series analysis method to analyze China's GDP, and through its prediction, selects the appropriate forecasting method to forecast the GDP of China's Inner Mongolia Autonomous Region. By this, we use the stationary test.

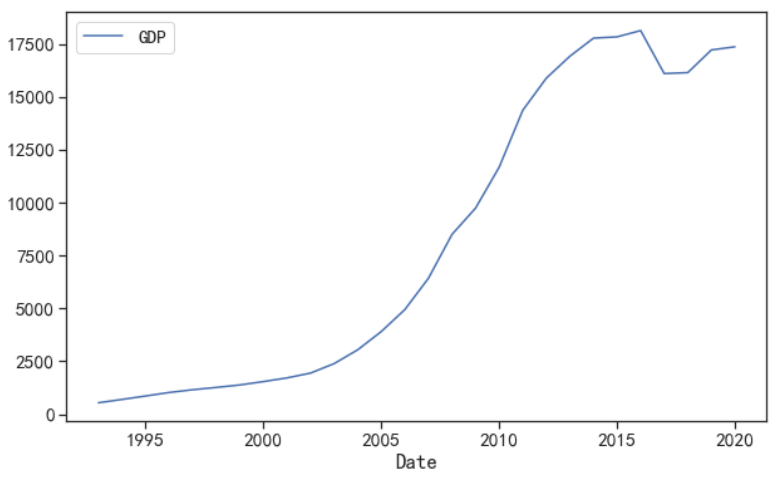

Figure 2 the GDP in inner Mongolia

Figure 2 illustrates the data of the Gross Domestic Product from 1993 to 2020 . We can clearly see from the figure that, in addition to the change in the calculation of GDP in 2017 and the impact of the new crown epidemic in 2020, the total GDP of the Inner Mongolia Autonomous Region has been growing steadily in recent years.

As can be seen from Table 1, we found that the $\mathrm{t}$ statistic value is more than -2.66 , so we believed that the sequence at this time was unstable.

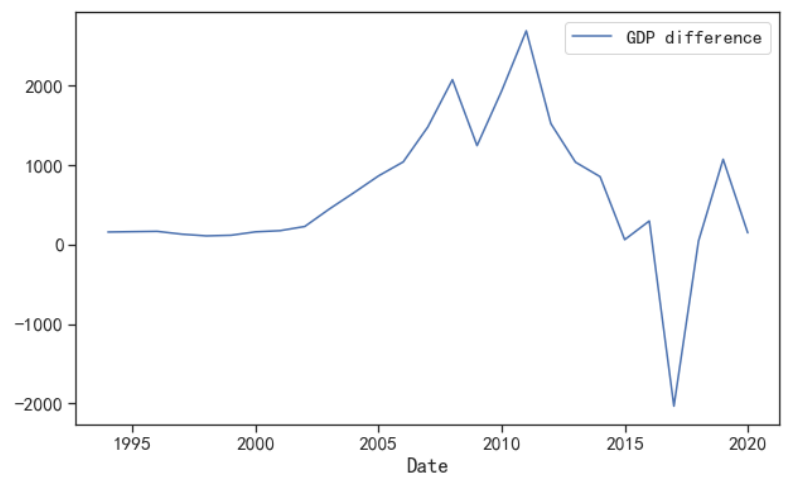

Figure 3 the GDP difference in inner Mongolia

In Figure 3, in order to evaluate and predict the GDP of the Inner Mongolia Autonomous Region, we have made a difference in the total annual GDP and tried to predict the stationarity. As can be seen from Table 1, we found that the t-statistic value is more than 2.67 , so we believed that the sequence at this time was unstable.

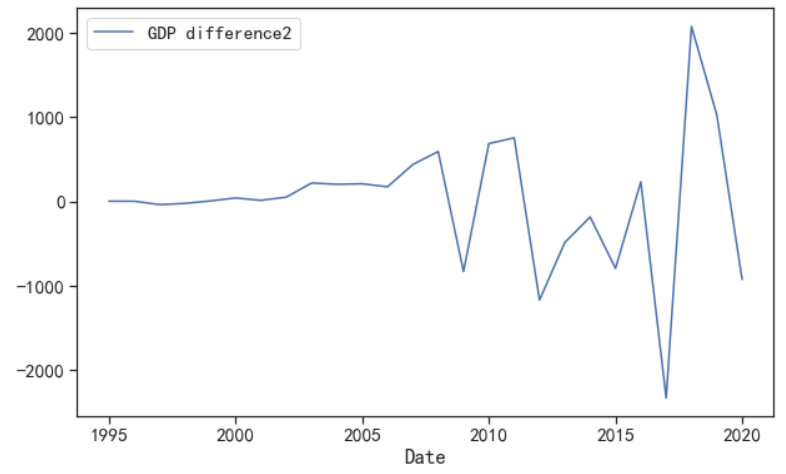

Figure 4 the second GDP difference in inner Mongolia

In Figure 4 we have carried out a second difference on the total GDP of the Inner Mongolia Autonomous Region to obtain more suitable data for stationarity testing. As can be seen from Table 1, we found that the t-statistic value is lower than -3.05 , so we believed that the sequence at this time was stable. Determinate that the final appropriate order $d$ of the $\operatorname{ARIMA}(p, d, q)$ model is equal to 2 .

Table 1 The results of the ADF unit root.

\begin{tabular}{|c|c|c|c|c|c|}
\hline Variables & $\begin{array}{c}\mathrm{t}- \\
\text { Statistic }\end{array}$ & $1 \%$ & $5 \%$ & $10 \%$ & Conclusion \\
\hline GDP & 4.06 & - & - & - & Unstable \\
\hline$\nabla$ GDP & 1.45 & - & - & - & Unstable \\
\hline$\nabla^{2} \mathrm{GDP}$ & -3.13 & - & - & - & Stable \\
& & 3.89 & 3.05 & 2.67 & \\
& & & & & \\
\hline
\end{tabular}




\subsection{Model Parameter Estimation}

Figure 5 illustrates that according to the AIC criterion, the AIC value is the smallest when $p=0$ and $\mathrm{q}=3$, which is the same as the result of image analysis. Therefore, the best fitting model of the time series $\nabla^{2}$ GDP is $\operatorname{ARIMA}(0,0,3)$, which means that the best fitting model of the time series $\operatorname{GDP}$ is $\operatorname{ARIMA}(0,2,3)$.

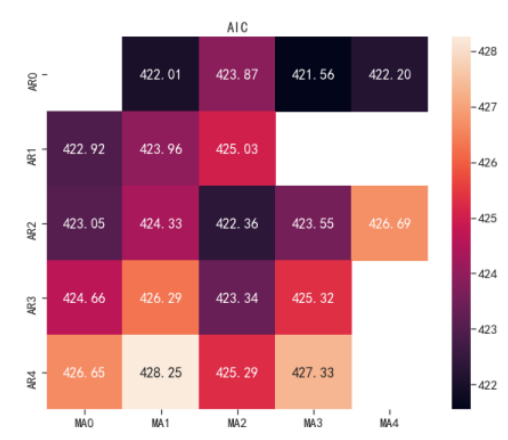

Figure 5 the second GDP difference in inner Mongolia

\subsection{White Noise Test}
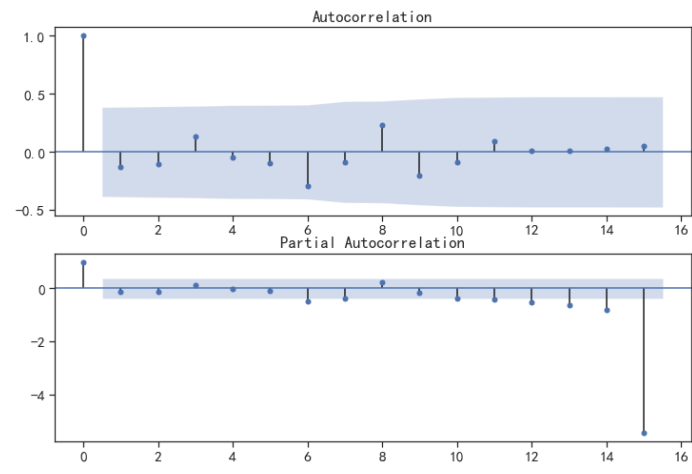

Figure 6 the Autocorrelation and Partial Autocorrelation of residual sequence.
Through the ljung-box test, determine whether there is a lagging correlation in the residual sequence. From Table 3, the p-values within 12 lags are all greater than 0.05 , so it can be considered that there is no significant sequence correlation in the residuals, which is a white noise sequence.

Table 2 The results of Ljung-Box test.

\begin{tabular}{|c|c|c|c|}
\hline lag & AC & Q & p-value \\
\hline 1.0 & -0.131924 & 0.506803 & 0.476526 \\
\hline 2.0 & -0.101987 & 0.822311 & 0.662884 \\
\hline 3.0 & 0.135215 & 1.401014 & 0.705297 \\
\hline 4.0 & -0.043548 & 1.463769 & 0.833039 \\
\hline 5.0 & -0.093899 & 1.769423 & 0.880040 \\
\hline 6.0 & -0.294215 & 4.920290 & 0.554076 \\
\hline 7.0 & -0.090132 & 5.231560 & 0.631728 \\
\hline 8.0 & 0.232307 & 7.414212 & 0.492671 \\
\hline 9.0 & -0.205407 & 9.221029 & 0.417127 \\
\hline 10.0 & -0.091280 & 9.600139 & 0.476246 \\
\hline 11.0 & 0.091277 & 10.004489 & 0.529983 \\
\hline 12.0 & 0.011052 & 10.010841 & 0.615010 \\
\hline
\end{tabular}

\subsection{Model Prediction}

We use the $\operatorname{ARIMA}(0,2,3)$ model above to forecast the GDP of Inner Mongolia Autonomous Region in next 5 years. The results are shown in the figure above. So far, the $\operatorname{ARIMA}(0,2,3)$ model has been established by smoothing the GDP data of Inner Mongolia Autonomous Region from 1993 to 2020. As table 3 said, we obtained the GDP forecast value of Inner Mongolia Autonomous Region in 2021-2025.

Table 3 The results of the predicted GDP value.

\begin{tabular}{|c|c|c|c|c|c|c|}
\hline \multirow{2}{*}{ Year } & \multicolumn{2}{|c|}{ Pessimistic scene } & \multicolumn{2}{c|}{ Reference scene } & \multicolumn{2}{c|}{ Optimistic scene } \\
\cline { 2 - 7 } & GDP & Increase rate & GDP & Increase rate & GDP & $17.1 \%$ \\
\hline 2021 & 17865.86 & $2.9 \%$ & 19097.71 & $9.9 \%$ & 20329.57 & $18.9 \%$ \\
\hline 2022 & 19289.62 & $8.0 \%$ & 21727.24 & $13.9 \%$ & 24164.86 & $17.9 \%$ \\
\hline 2023 & 20176.50 & $4.6 \%$ & 24331.39 & $12.0 \%$ & 28486.29 & $18.7 \%$ \\
\hline 2024 & 20058.54 & $-0.6 \%$ & 26935.54 & $10.7 \%$ & 33812.54 & $17.7 \%$ \\
\hline 2025 & 19293.69 & $-4.0 \%$ & 29539.69 & $9.7 \%$ & 39785.70 & \\
\hline
\end{tabular}




\section{CONCLUSION}

In conclusion, the $\operatorname{ARIMA}(0,2,3)$ model is made based on the GDP data of Inner Mongolia Autonomous Region from 1993 to 2020 . The adaptability test of the model proposes that the model can be used to predict the GDP from 2021-2025 of Inner Mongolia Autonomous Region, and can provide consult for Inner Mongolia Autonomous Region to make a series of economic planning.

According to the 14 th Five-Year Plan of Inner Mongolia, the average annual growth of regional GDP will reach $4.3 \%$. According to our forecast results, we can find that due to the impact of the COVID-19 in 2020 , it will only increase by $0.9 \%$ over 2019 . However, according to the results of previous years, it is found that the GDP in 2019 has increased by $5.2 \%$, and the GDP in 2018 has increased by $5.3 \%$. The GDP index growth of Inner Mongolia Autonomous Region is basically qualified. Although according to our forecast, the GDP in 2021 will be about 19097.71, which is an increase of about $9.9 \%$ over 2020, so through the continuous efforts and development of the Inner Mongolia Autonomous Region government, it must be restored to the state before the epidemic. Obviously, the Inner Mongolia government can also adopt this forecast, and it can use these data to adjust the economic direction and further promote growth.

The government need implement the innovationdriven development strategy to comprehensively shape new development momentum; maintain the strategic determination to strengthen the construction of ecological civilization, and build an important ecological safety barrier in northern China; optimize the regional economic layout, promote regional coordinated development; promote the high-quality development of the real economy, and build green characteristics Advantageous modern industrial system; accelerating digital development to create a new engine for highquality development; deepening reforms in key areas based on the business environment to enhance highquality development motivation; integrating into the domestic cycle and smoothing the domestic and international dual cycles, and building China's opening to the north is important Bridgehead; Promote the development of concentrated and intensive contracts, and build a new urbanization pattern with people as the core; comprehensively promote rural revitalization and accelerate the modernization of rural pastoral areas; build a modern infrastructure system to enhance the ability to guarantee high-quality development; improve the quality of the population and promote the all-round development of people Enhance people's livelihood and well-being, enhance the level of co-construction, cogovernance and sharing; prosper and develop cultural undertakings and cultural industries to meet the cultural needs of the people; build a sense of the Chinese nation's community and solidly promote national unity; build a higher level of peace in Inner Mongolia and strengthen the security of the northern border Stabilize barriers and other content.

\section{REFERENCES}

[1] Huang G, Jiang Y. Urbanization and Socioeconomic Development in Inner Mongolia in 2000 and 2010: A GIS Analysis. Sustainability. 2017; 9(2):235. https://doi.org/10.3390/su9020235

[2] HAN Weihong, PAN Lingling, YANG Xinjiletua.Research on the Economic Impact of New Energy Fiscal and Tax Policies Based on CGE Model: A Case from Inner Mongolia. E3S Web Conf. 2018.

[3] Yuanning Hu, Jikun Huang, Lingling Hou,Impacts of the Grassland Ecological Compensation Policy on Household Livestock Production in China: An Empirical Study in Inner Mongolia,Ecological Economics,2019.

[4] Rehan Jamil,Hydroelectricity consumption forecast for Pakistan using ARIMA modeling and supplydemand analysis for the year 2030, Renewable Energy,2020.

[5] Yuping Bai, Wenxuan Wang, Yecui Hu, Zehao Wang,County-level estimates of population and economic scenarios under the shared socioeconomic pathways: A case study in Inner Mongolia, China,Physics and Chemistry of the Earth, 2021.

[6] S. Siami-Namini, N. Tavakoli and A. Siami Namin, "A Comparison of ARIMA and LSTM in Forecasting Time Series," 2018 17th IEEE International Conference on Machine Learning and Applications (ICMLA), 2018, pp. 1394-1401, doi: 10.1109/ICMLA.2018.00227.

[7] Domenico Benvenuto, Marta Giovanetti, Lazzaro Vassallo, Silvia Angeletti, Massimo Ciccozzi,Application of the ARIMA model on the COVID-2019 epidemic dataset,Data in Brief,2020.

[8] Wang, Ya-wen and Shen, Zhong-zhou and Jiang, Yu, Comparison of ARIMA and $\operatorname{GM}(1,1)$ models for prediction of hepatitis $\mathrm{B}$ in China, PLOS ONE,2018.

[9] Zou, S. (2020) Research on GDP Forecast of Ji'an City Based on ARIMA Model. Open Journal of Social Sciences, 8, 353-365. doi: $10.4236 /$ jss.2020.812029. 